\title{
Comparison of CT Severity Index and Modified CT Severity Index in the Clinical Severity Assessment of Acute Pancreatitis
}

\author{
Piyaporn Apisarnthanarak, M.D. ${ }^{1}$, Pattira Boonsri, M.D. ${ }^{1}$, Voraparee Suvannarerg, M.D. ${ }^{1}$, \\ Walailak Chaiyasoot, M.D., Supot Pongprasobchai, M.D. ${ }^{2}$, Anucha Apisarnthanarak, M.D. ${ }^{3}$
}

'Department of Radiology, Faculty of Medicine, Siriraj Hospital, Mahidol University, Bangkok Noi, Bangkok 10700, Thailand.

${ }^{2}$ Department of Medicine, Faculty of Medicine, Siriraj Hospital, Mahidol University, Bangkok Noi, Bangkok 10700, Thailand.

${ }^{3}$ Division of Infectious Diseases, Thammasat University Hospital, Klong Luang, Pathumthani 12120, Thailand.

Received 13 August 2021 • Revised 10 October 2021 • Accepted 21 October 2021 • Published online 26 November 2021

\begin{abstract}
:
Objective: To compare the computed tomography severity index (CTSI) and the modified computed tomography severity index (MCTSI) in the clinical severity assessment of acute pancreatitis.

Material and Methods: This retrospective cohort study comprised acute pancreatitis patients who underwent contrastenhanced abdominal computed tomography (CT) scans within 4 weeks after clinical onset. Two experienced abdominal radiologists, blinded to the clinical outcome, independently reviewed the CT images and retrospectively scored them using CTSI and MCTSI. Any discrepancies were resolved by a consensus review. The clinical severity assessment of each participant was categorized by the determinant-based classification of acute pancreatitis severity. The correlations of CTSI and MCTSI with the clinical severity assessment were analyzed.

Results: This cohort study consisted of 40 participants - 28 of them were men (70.0\%) — with a mean age of 59.3 years. They were clinically divided into mild, moderate, severe, and critical groups comprising $11(27.5 \%), 16(40.0 \%), 7$ (17.5\%), and $6(15.0 \%)$ participants, respectively. Due to the small number of patients in the severe and critical groups, we combined these into a single severe category (13 patients, 32.5\%). The CTSI and MCTSI scores showed moderate and fair agreement with the clinical severity assessment. A trend linking poor clinical outcome with high CTSI/MCTSI scores (moderate and severe groups) more commonly than low scores (mild group) was observed. There was a very strong agreement between CTSI and MCTSI $\left(r_{s}=0.97\right)$.
\end{abstract}

Contact: Anucha Apisarnthanarak, M.D.

Division of Infectious Diseases, Thammasat University Hospital, Klong Luang, Pathumthani 12120, Thailand.

E-mail: anapisarn@yahoo.com

(c) 2021 JHSMR. Hosting by Prince of Songkla University. All rights reserved.

This is an open access article under the CC BY-NC-ND license

(http://www.jhsmr.org/index.php/jhsmr/about/editorialPolicies\#openAccessPolicy).
J Health Sci Med Res 2022;40(4):425-435 doi: 10.31584 /jhsmr.2021854 www.jhsmr.org 
Conclusion: CTSI and MCTSI showed a moderate and fair agreement, respectively, with the clinical severity assessment. Compared to low scores, a poor clinical outcome was more often associated with high CTSIMCTSI scores.

Keywords: acute pancreatitis, computed tomography severity index (CTSI), determinant-based classification of acute pancreatitis severity, modified computed tomography severity index (MCTSI)

\section{Introduction}

Acute pancreatitis is an acute inflammation of the pancreas with a variable involvement of peripancreatic tissues, adjacent organs, and vital body systems (e.g., cardiovascular, respiratory, and renal systems). It is frequently presented as an emergent abdominal condition, which needs timely diagnosis and prompt treatment to prevent possible complications. ${ }^{1-3}$ About eighty percent of patients with acute pancreatitis have mild symptoms, experience self-limited courses of the disease, and recover with conservative treatment without any complications. A minority, however, have severe symptoms with local and systemic complications, causing a mortality rate of up to twenty-four percent. ${ }^{4}$ These cases need surgery or percutaneous intervention for treatment.

The clinical severity of acute pancreatitis can be categorized by many methods, such as the revised Atlanta classification of acute pancreatitis ${ }^{1}$, which categorizes cases as mild, moderately severe, or severe acute pancreatitis, depending on the presence and duration of organ failure, and the presence of local or systemic complications. The determinant-based classification of acute pancreatitis severity ${ }^{5}$ is another method for the clinical severity assessment of acute pancreatitis. It is based on the presence or absence of pancreatic or peripancreatic necrosis, superimposed infection, and the duration of organ failure (transient or persistent). According to this method, the severity of acute pancreatitis is categorized as mild, moderate, severe, or critical acute pancreatitis (Table 1). Organ failure is defined as the failure of at least one of the following three systems: cardiovascular, respiratory, and renal systems, according to the sepsis-related organ failure assessment (SOFA) score. ${ }^{6}$ If the organ failure persists for longer than 48 hours, it is classified as persistent organ failure.

In addition, the severity assessment of acute pancreatitis can be conducted using computed tomography (CT) findings. The CT severity index (CTSI), for example, proposed by Balthazar et al. ${ }^{7,8}$ (Table 2), has been well accepted as an accurate method for the severity assessment of acute pancreatitis, and it is well related with clinical outcome. It focuses on the degree of pancreatic inflammation and necrosis and defines its result based on a 10-point ordinal scale, categorized as mild (score 0-3), moderate (score 4-6), and severe (score 7-10) acute pancreatitis. It predicts the severity, morbidity, and mortality

Table 1 Determinant-based classification of acute pancreatitis (AP) severity ${ }^{5}$

\begin{tabular}{llll} 
& Mild AP & Moderate AP & Severe AP \\
\hline Peri) Pancreatic necrosis & No & Sterile & Infected \\
& AND & AND/OR & OR Infected \\
Organ failure & No & Transient & Persistent \\
\hline
\end{tabular}


of acute pancreatitis patients well, but it is limited in its ability to predict extrapancreatic complications $s^{9,10}$ and organ failure. ${ }^{11}$

The modified CT severity index (MCTSI) was developed in 2004 by Mortele et al. ${ }^{12}$ (Table 3) with the addition of extrapancreatic complications as keys for the MCTSI calculation, with the aim of improving the accuracy of predicting the severity, morbidity, and mortality of acute pancreatitis patients. They reduced the score of pancreatic necrosis since they acknowledged that there was no significant difference in morbidity and mortality between patients with thirty to fifty percent pancreatic necrosis and patients with more than fifty percent pancreatic necrosis. MCTSI also employs a 10-point metric scale that categorizes mild (score 0-2), moderate (score 4-6,) and severe (score 8-10) acute pancreatitis.

Mortele et al. ${ }^{12}$ reported that $\mathrm{MCTSI}$ is correlated more closely with the patient outcome than CTSI; meanwhile, Bollen et al. ${ }^{13}$ reported no significant differences between the two in assessing the severity of acute pancreatitis. The purpose of the present study was to compare CTSI and MCTSI in assessing the severity of acute pancreatitis by correlating their scores with clinical severity assessments using the determinant-based classification of acute pancreatitis severity.

Table 2 Computed tomography severity index (CTSI)

\begin{tabular}{ll}
\hline Prognostic indicator & Points \\
\hline Pancreatic inflammation & 0 \\
Normal pancreas & 1 \\
Focal or diffuse enlargement of pancreas & 2 \\
Intrinsic pancreatic abnormalities with inflammatory changes in peripancreatic fat & 3 \\
Single, ill-defined fluid collection or phlegmon & 4 \\
Two or more poorly-defined collections or presence of gas in or adjacent to pancreas \\
Pancreatic necrosis \\
None \\
$\leq 30 \%$ \\
$>30-50 \%$ \\
$>50 \%$
\end{tabular}

Table 3 Modified computed tomography severity index (MCTSI) ${ }^{12}$

\begin{tabular}{l}
\hline Prognostic indicator \\
\hline Pancreatic inflammation \\
Normal pancreas \\
Intrinsic pancreatic abnormalities with or without inflammatory changes in peripancreatic fat \\
Pancreatic or peripancreatic fluid collection or peripancreatic fat necrosis \\
Pancreatic necrosis \\
None \\
$\leq 30 \%$ \\
$>30 \%$ \\
Extrapancreatic complications (one or more of the following: pleural effusion, ascites, vascular complications, \\
parenchymal complications, or gastrointestinal tract involvement) \\
\hline
\end{tabular}




\section{Material and Methods}

This was a retrospective single-center study from a 2,220-bed university hospital in Bangkok, Thailand. The hospital's institutional review board approved it with a waiver for the need of a written informed consent. The included participants were acute pancreatitis patients, who had contrast-enhanced abdominal CT scans performed at our institution between December 2011 and December 2014 and within 30 days after the onset of acute pancreatitis. Patients with underlying chronic pancreatitis, poor-quality CT images that were not suitable for interpretation, and no available medical history for review were excluded from the study.

The clinical and admission information of each patient was recorded by one of the investigators (PB). The collected clinical information included gender, age, underlying disease, and history of alcohol abuse. The recorded admission information included the length of hospital/intensive care unit (ICU) stay, the patient condition on the discharge date, the development of infected pancreatic necrosis and organ failure, treatment details (medical treatment, surgery, percutaneous intervention), and the severity of disease graded in accordance with the determinant-based classification of acute pancreatitis severity ${ }^{5}$ (Table 1).

The CT scans were performed via three 64-slice CT scanners (LightSpeed VCT, GE Healthcare, United States; Discovery CT750 High Definition, GE Healthcare, United States; and SOMATOM Definition Dual Source, Siemens, Germany). The scan area covered the upper abdomen as a minimum. The slice collimations were 1.25 millimeters $(\mathrm{mm})$ with a reconstruction at $7.0 \mathrm{~mm}$ for the LightSpeed VCT and Discovery CT750 High Definition system, and 1.5 $\mathrm{mm}$ with a reconstruction at $7.0 \mathrm{~mm}$ for the SOMATOM Definition Dual Source system. All subjects underwent noncontrast and contrast enhanced phase scans before and after an intravenous injection of $80-100$ milliliters $(\mathrm{mL})$ of nonionic iodinated contrast agent followed by $20-40 \mathrm{~mL}$ of normal saline through a power injector with a rate of 3 $\mathrm{mL} /$ second. A portovenous phase scan with an 80-second delay was obtained in all participants. Some participants had an additional pancreatic phase scan at a 40-second delay or a delayed phase scan at a 5-minute delay, depending on clinical indication.

The CT images of each participant were independently and retrospectively assessed on the Picture Archiving and Communication System (PACS) by two fellowship-trained abdominal radiologists (PA and VS with 20 and 11 years of clinical experience in the evaluation of abdominal CT scans, respectively). They knew that the participants were acute pancreatitis patients but were blinded to their clinical outcome. They identified the etiology of acute pancreatitis when possible. The severity of acute pancreatitis was graded via both CTSI and MCTSI. Any discrepancies were reassessed by a consensus review between the two radiologists.

Descriptive statistics, number (\%) and mean (range), were used to summarize the data. The duration of time was presented as a median (range). The agreement of the severity of acute pancreatitis according to CTSI and MCTSI with the clinical severity assessment was calculated using the weighted Cohen's Kappa (Kw $\leq$ 0: no agreement; 0.010.20: non to slight agreement; 0.21-0.40: fair agreement; 0.41-0.60: moderate agreement; 0.61-0.80: substantial agreement; and 0.81-1.00: almost perfect agreement) with a 95\% confidence interval $(\mathrm{Cl})$. The Kruskal-Wallis test and Fisher's exact test were used for comparison between groups. The correlation between CTSI and MCTSI was assessed via Spearman's rank correlation coefficient (rho or $r_{s}=0-0.09$ : no agreement; 0.10-0.29: poor agreement; 0.30-0.59: fair agreement; 0.60-0.79: moderate agreement; 0.80-0.99: very strong agreement; and 1.00: perfect agreement). ${ }^{14}$ 
All the statistical analyses were obtained using PASW Statistics 18 (SPSS Inc., Chicago, IL, USA) and the MedCalc Statistical Software Version 18.2.1 (MedCalc Software bvba, Ostend, Belgium). A 2-sided p-value of $\leq$ 0.050 was considered to indicate statistical significance.

\section{Results}

Following the described inclusion and exclusion criteria, we initially enrolled 43 patients with acute pancreatitis, who had undergone a contrast-enhanced CT scan within 30 days after the onset of acute pancreatitis. Of these, 3 had underlying chronic pancreatitis and were excluded from the study population. The remaining 40 patients were recruited and constituted the final study cohort. There were 28 men (70.0\%) and 12 women (30.0\%) with a mean age of 59.3 years (range 27-97 years). The median duration between the onset of acute pancreatitis and CT scan was 6 days (range 1-17 days). Ten (25.0\%) participants had less than 72 hours of clinical onset before the CT scan. The most common etiology of acute pancreatitis identified by the CT scan was a biliary tract stone (18 patients, $45.0 \%$ ). Eleven patients (27.5\%) had a history of alcohol abuse.

The participants' median length of hospital stay was 13 days (range $3-147$ days). Nine patients (22.5\%) were admitted to the ICU with a median length of ICU stay of 5 days (range 1-92 days). Four patients (10.0\%) passed away. The rest (36 patients, 90.0\%) were clinically improved on the discharge date. Three patients $(7.5 \%)$ underwent CT-guided percutaneous drainage, one patient $(2.5 \%)$ underwent angiography with embolization, and one patient (2.5\%) underwent both CT-guided percutaneous drainage and open necrosectomy. The details of the patients' demographic data are displayed in Table 4.
Table 4 Demographic data of study population

\begin{tabular}{ll}
\hline Demographic data & $\begin{array}{l}\text { Number } \\
\text { of patients (\%) }\end{array}$ \\
\hline Etiologies of acute pancreatitis & $18(45.0)$ \\
Biliary tract stone & $4(10.0)$ \\
Alcohol & $4(10.0)$ \\
Hypertriglyceridemia & $8(20.0)$ \\
Idiopathic cause & $2(5.0)$ \\
Pancreatic tumor & $4(10.0)$ \\
Post ERCP & $11(27.5)$ \\
History of alcohol abuse & \\
Intervention & $4(10.0)$ \\
$\quad$ CT-guided percutaneous drainage* & $1(2.5)$ \\
Angiography with embolization & $1(2.5)$ \\
Surgery (open necrosectomy)* & \\
\hline
\end{tabular}

$\mathrm{ERCP}=$ endoscopic retrograde cholangiopancreatography, $\mathrm{CT}=$ computed tomography

*3 patients underwent CT-guided percutaneous drainage, and 1 patient underwent both CT-guided percutaneous drainage and open necrosectomy.

Based on the determinant-based classification of acute pancreatitis severity ${ }^{5}$, the participants were categorized as having mild, moderate, severe, or critical acute pancreatitis, with 11 (27.5\%), 16 (40.0\%), 7 (17.5\%), and $6(15.0 \%)$ patients in each group, respectively. Due to the small number of patients in the severe and critical groups, we combined them into a single group, i.e., the severe category (13 patients, $32.5 \%$ ).

The most common CT finding in this study was edematous pancreas (38 patients, 95.0\%), followed by peripancreatic fat stranding (37 patients, 92.5\%), and peripancreatic fluid (31 patients, 77.5\%) (Figure 1).

Pancreatic and/or peripancreatic necrosis was seen in $24(60.0 \%)$ patients, which was categorized as sterile necrosis (20 patients) and infected necrosis (4 patients) by the presence of internal air (Figure 2). All four infected 


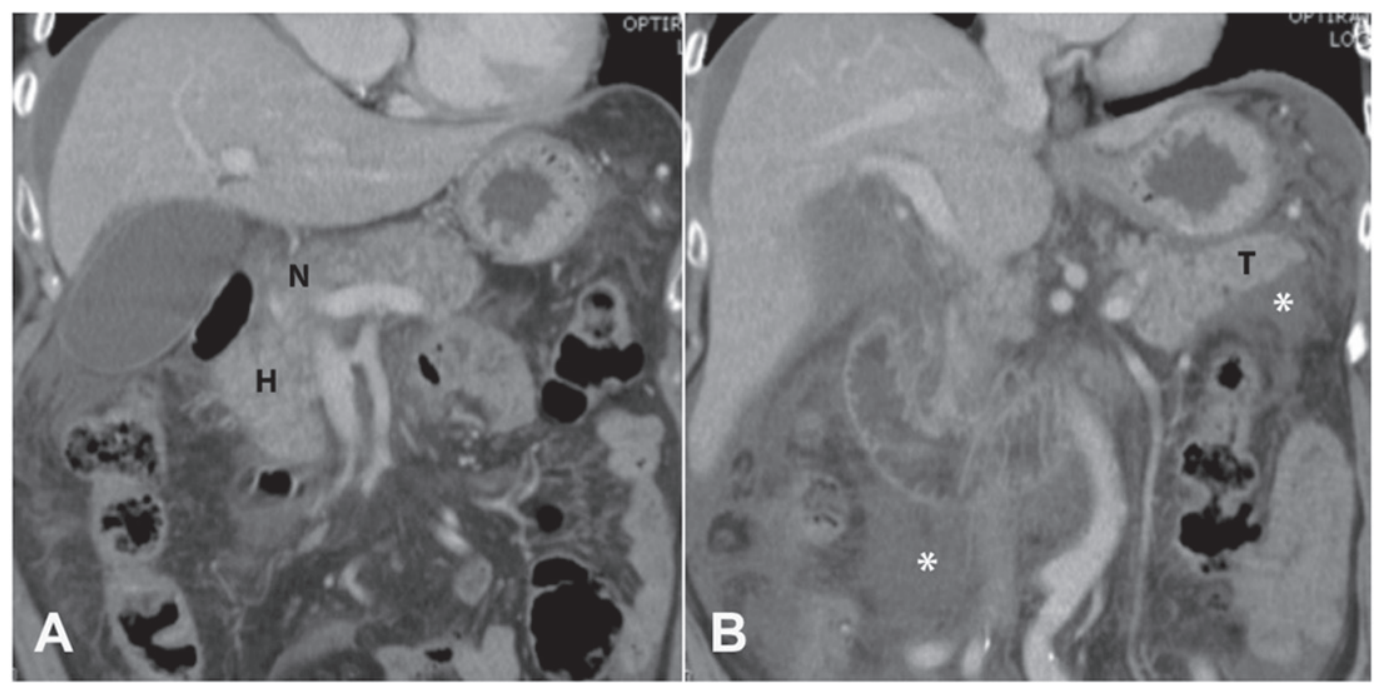

Figure 1 Interstitial edematous pancreatitis with acute peripancreatic fluid collections (APFCs). Coronal contrast-enhanced CT images of a 61-year-old male with acute pancreatitis ( $A$ and $B$, from the anterior to the posterior) showing an edematous pancreas, more prominent at the head $(\mathrm{H})$ and neck $(\mathrm{N})$ than the tail $(\mathrm{T})$, without evidence of pancreatic necrosis. Peripancreatic fluid is clearly visualized (asterisks in 1B). Using CTSI and MCTSI, the patient was assessed to have moderate acute pancreatitis (CTSI=4 and MCTSI=6).

cases were proven; 3 patients underwent CT-guided percutaneous drainage, and another underwent both CT-guided percutaneous drainage and subsequent open necrosectomy.

\section{CTSI (Table 5)}

In terms of severity assessment by CTSI, the patients were categorized as having mild (score 0-3), moderate (score 4-6), or severe (score 7-10) acute pancreatitis, with $13(32.5 \%), 19(47.5 \%)$, and $8(20.0 \%)$ patients in each group, respectively.

There was agreement on the severity of acute pancreatitis between CTSI and clinical assessment based on the determinant-based classification of acute pancreatitis severity for 9 of $13(69.2 \%)$ patients in the mild group, 8 of $19(42.1 \%)$ patients in the moderate group, and 3 of 8 $(37.5 \%)$ patients in the severe group. Overall, moderate agreement between CTSI and clinical severity assessment was found by the weighted Cohen's Kappa analysis (Kw $=0.49,95 \% \mathrm{Cl}=0.29-0.70)$.

The mean length of hospital stay in the mild, moderate, and severe groups categorized according to CTSI was 13,11 , and 20 days, respectively. One (7.7\%) patient in the mild group, 6 (31.6\%) patients in the moderate group, and $2(25.0 \%)$ patients in the severe group were admitted to the ICU. Of the 4 patients with infected necrosis, 2 were in the moderate group, and the other 2 were in the severe group. Organ failure was mostly found in the moderate group (9 patients, 47.4\%). Moreover, the 4 patients who passed away were also in the moderate group.

Angiography with embolization and open necrosectomy were performed only in the severe group. Two patients in the moderate group, and 2 patients in the severe group underwent CT-guided percutaneous drainage. 


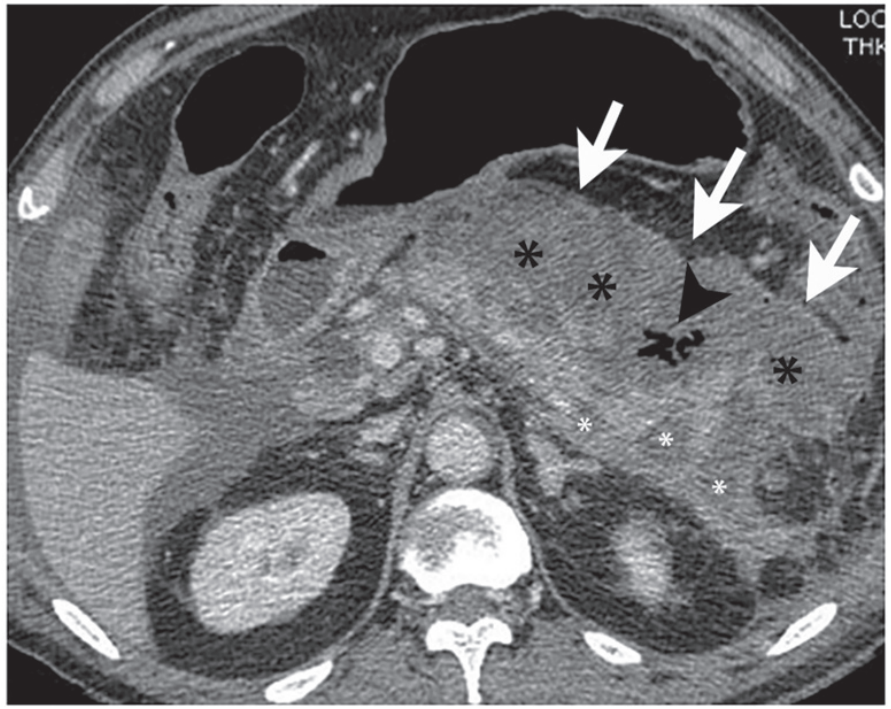

Figure 2 Acute necrotizing pancreatitis with infected acute necrotic collection (ANC). Axial contrast-enhanced CT image of a 77-year-old female with acute pancreatitis showing pancreatic necrosis (white asterisks) and peripancreatic fat necrosis (black asterisks). Small amount of air (black arrowhead) within the collection (white arrows) proposes the diagnosis of infected ANC.

Using CTSI and MCTSI, the patient was assessed as having severe acute pancreatitis (CTSI=10 and $\mathrm{MCTSI}=10)$.

Table 5 Correlation between computed tomography severity index (CTSI) and patient outcome

\begin{tabular}{|c|c|c|c|c|c|}
\hline \multirow[b]{2}{*}{ Clinical parameter } & \multirow[b]{2}{*}{ Total } & \multicolumn{3}{|c|}{ CTSI } & \multirow[b]{2}{*}{$\mathrm{p}$-value } \\
\hline & & $\begin{array}{l}\text { Mild } \\
(0-3)\end{array}$ & $\begin{array}{l}\text { Moderate } \\
(4-6)\end{array}$ & $\begin{array}{l}\text { Severe } \\
(7-10)\end{array}$ & \\
\hline No. of patients & 40 & 13 & 19 & 8 & \\
\hline $\begin{array}{l}\text { No. of patients according to clinical } \\
\text { severity }\end{array}$ & & & & & $\begin{array}{l}\mathrm{Kw}=0.49 \\
95 \% \mathrm{Cl}(0.29,0.70)\end{array}$ \\
\hline Mild & 11 & $9(69.2 \%)$ & $2(10.5 \%)$ & $0(0.0 \%)$ & \\
\hline Moderate & 16 & $3(23.1 \%)$ & $8(42.1 \%)$ & $5(62.5 \%)$ & \\
\hline Severe & 13 & $1(7.7 \%)$ & $9(47.4 \%)$ & $3(37.5 \%)$ & \\
\hline Median (range) length of hospital stay (days) & & $13(5-29)$ & $11(3-147)$ & $20(8-110)$ & $0.121^{a}$ \\
\hline No. of patients admitted to ICU & 9 & $1(7.7 \%)$ & $6(31.6 \%)$ & $2(25.0 \%)$ & $0.327^{\mathrm{b}}$ \\
\hline No. of patients with infected necrosis & 4 & $0(0.0 \%)$ & $2(10.5 \%)$ & $2(25.0 \%)$ & $0.172^{b}$ \\
\hline No. of patients with organ failure & 17 & $3(23.1 \%)$ & $9(47.4 \%)$ & $5(62.5 \%)$ & $0.200^{\mathrm{b}}$ \\
\hline $\begin{array}{l}\text { No. of patients who underwent } \\
\text { procedures }\end{array}$ & & & & & $0.076^{b}$ \\
\hline Angiography with embolization & 1 & $0(0.0 \%)$ & $0(0.0 \%)$ & $1(12.5 \%)$ & \\
\hline CT-guided percutaneous drainage ${ }^{*}$ & 4 & $0(0.0 \%)$ & $2(10.5 \%)$ & $2(25.0 \%)$ & \\
\hline Open necrosectomy* & 1 & $0(0.0 \%)$ & $0(0.0 \%)$ & $1(12.5 \%)$ & \\
\hline No. of deaths & 4 & $0(0.0 \%)$ & $4(21.1 \%)$ & $0(0.0 \%)$ & $0.120^{b}$ \\
\hline
\end{tabular}

No.=number, ICU=intensive care unit, CT=computed tomography, Kw=weighted Cohen's Kappa, 95\% Cl=95\% confidence interval ${ }^{a}$ Kruskal-Wallis test, ${ }^{\mathrm{b}}$ Fisher's exact test.

*3 patients underwent CT-guided percutaneous drainage, and 1 patient underwent both CT-guided percutaneous drainage and open necrosectomy. 


\section{MCTSI (Table 6)}

Concerning the severity assessment via MCTSI, the patients were categorized as having mild (score 0-2), moderate (score 4-6), or severe (score 8-10) acute pancreatitis, with $2(5.0 \%), 22(55.0 \%)$, and $16(40.0 \%)$ patients in each group, respectively.

Agreement on the severity of acute pancreatitis according to MCTSI and clinical assessment based on a determinant-based classification of acute pancreatitis severity was reached among 2 of $2(100.0 \%)$ patients in the mild group, 5 of $22(22.7 \%)$ patients in the moderate group, and 5 of $16(31.3 \%)$ patients in the severe group. Overall, the weighted Cohen's Kappa indicated fair agreement between MCTSI and clinical severity assessment $(\mathrm{K} w=0.31$, $95 \% \mathrm{Cl}=0.11-0.51)$.
The mean length of hospital stay in the mild, moderate and severe groups categorized according to MCTSI was 14, 10, and 15 days, respectively. Six (27.3\%) patients in the moderate group and $3(18.8 \%)$ patients in the severe group were admitted to the ICU. Four patients had infected necrosis; 1 was in the moderate group, and the other 3 were in the severe group. Organ failure was found only in the moderate (10 patients, $45.5 \%$ ) and severe (7 patients, 43.8\%) groups. Four patients who passed away were in the moderate group.

Angiography with embolization and necrosectomy were performed only in the severe group. One patient in the moderate group, and 3 patients in the severe group underwent CT-guided percutaneous drainage.

Table 6 Correlation between modified computed tomography severity index (MCTSI) and patient outcome

\begin{tabular}{|c|c|c|c|c|c|}
\hline \multirow[b]{2}{*}{ Clinical parameter } & \multirow[b]{2}{*}{ Total } & \multicolumn{3}{|c|}{ MCTSI } & \multirow[b]{2}{*}{$\mathrm{p}$-value } \\
\hline & & $\begin{array}{l}\text { Mild } \\
(0-2)\end{array}$ & $\begin{array}{l}\text { Moderate } \\
(4-6)\end{array}$ & $\begin{array}{l}\text { Severe } \\
(8-10)\end{array}$ & \\
\hline No. of patients & 40 & 2 & 22 & 16 & \\
\hline $\begin{array}{l}\text { No. of patients according to clinical } \\
\text { severity }\end{array}$ & & & & & $\begin{array}{l}\mathrm{Kw}=0.31 \\
95 \% \mathrm{Cl}(0.11,0.51)\end{array}$ \\
\hline Mild & 11 & $2(100.0 \%)$ & $9(40.9 \%)$ & $0(0.0 \%)$ & \\
\hline Moderate & 16 & $0(0.0 \%)$ & $5(22.7 \%)$ & $11(68.8 \%)$ & \\
\hline Severe & 13 & $0(0.0 \%)$ & $8(36.4 \%)$ & $5(31.3 \%)$ & \\
\hline Median (range) length of hospital stay (days) & & $14(5-22)$ & $10(3-59)$ & $15(4-147)$ & $0.283^{\mathrm{a}}$ \\
\hline No. of patients admitted to ICU & 9 & $0(0.0 \%)$ & $6(27.3 \%)$ & $3(18.8 \%)$ & $0.825^{\mathrm{b}}$ \\
\hline No. of patients with infected necrosis & 4 & $0(0.0 \%)$ & $1(4.5 \%)$ & $3(18.8 \%)$ & $0.427^{\mathrm{b}}$ \\
\hline No. of patients with organ failure & 17 & $0(0.0 \%)$ & $10(45.5 \%)$ & $7(43.8 \%)$ & $0.662^{b}$ \\
\hline $\begin{array}{l}\text { No. of patients who underwent } \\
\text { procedures }\end{array}$ & & & & & $0.424^{b}$ \\
\hline Angiography with embolization & 1 & $0(0.0 \%)$ & $0(0.0 \%)$ & $1(6.3 \%)$ & \\
\hline CT-guided percutaneous drainage* & 4 & $0(0.0 \%)$ & $1(4.5 \%)$ & $3(18.8 \%)$ & \\
\hline Open necrosectomy* & 1 & $0(0.0 \%)$ & $0(0.0 \%)$ & $1(6.3 \%)$ & \\
\hline No. of deaths & 4 & $0(0.0 \%)$ & $4(18.2 \%)$ & $0(0.0 \%)$ & $0.211^{b}$ \\
\hline
\end{tabular}

No.=number, ICU=intensive care unit, CT=computed tomography, Kw=weighted Cohen's Kappa, $95 \% \mathrm{Cl}=95 \%$ confidence interval ${ }^{a}$ Kruskal-Wallis test, ${ }^{\mathrm{b}}$ Fisher's exact test

*3 patients underwent CT-guided percutaneous drainage, and 1 patient underwent both CT-guided percutaneous drainage and open necrosectomy. 
A very strong agreement, assessed via Spearman's rank correlation coefficient (rho or $r_{s}=0.97, p$-value $<0.001$ ), was detected between CTSI and MCTSI.

\section{Discussion}

CT scan is the imaging modality of choice for acute pancreatitis. It provides an accurate diagnosis, identifies possible causes, discloses severity, shows complications, guides appropriate treatment, and can also be performed for follow-up after treatment. CTSI ${ }^{7,8}$ and $\mathrm{MCTSI}^{12,13}$ are CT scoring systems for the assessment of the severity of acute pancreatitis that have been well-accepted and have shown to relate well with clinical outcome. The purpose of this study was to assess the accuracy of these scoring systems in the assessment of the severity of acute pancreatitis by correlating their scores with clinical severity assessment using the determinant-based classification of acute pancreatitis severity.

Here, albeit less than expected, we found a moderate and fair agreement between CTSI and MCTSI scores and the determinant-based classification of acute pancreatitis severity, respectively. This lower-than-expected level of agreement was possibly related to some differences between our study design and those of prior studies. Mortele et al. ${ }^{12}$ used the length of hospital/1CU stay, the need for surgical or percutaneous procedures, the incidence of pancreatic infection, and the development of organ failure as clinical outcomes. Bollen et al. ${ }^{13}$ used the same parameters with the addition of mortality rate and the APACHE II score as clinical outcomes. Our study used almost the same parameters as Bollen et al., but we replaced the APACHE II score with the determinant-based classification of acute pancreatitis severity. Nevertheless, our study showed a trend of poor clinical outcome in cases with a high CTSI/MCTSI score (moderate and severe groups) compared to those with lower scores (mild group). These included the number of patients admitted to the ICU, number of patients with infected pancreatic necrosis, number of patients with organ failure, the need for procedures (surgery or intervention), and mortality rate. Regarding the length of hospital stay, the moderate group evaluated via CTSI and MCTSI showed the shortest length of hospital stay compared to the mild and severe groups. The only explanation we could offer for this finding was that two of the four patients who passed away had short durations of hospital stay ( 3 and 4 days), and they belonged to the moderate group. This impacted the accuracy of using the length of hospital stay as a clinical outcome of acute pancreatitis.

There was a very strong agreement between CTSI and MCTSI in relation to the severity assessment of acute pancreatitis. This could be easily explained since the main indicators of both scoring systems are the signs of pancreatic inflammation and the degree of pancreatic necrosis. A difference between these 2 scoring systems is that MCTSI partly focuses on extrapancreatic complications. Nevertheless, since the score for extrapancreatic complications is only 0-2 points, it could not make a significant difference between the scores yielded by the two systems.

The most common etiology of acute pancreatitis in this study was biliary tract stone, the same as reported by Bollen et al. ${ }^{13}$ Although the median duration between the onset of acute pancreatitis and the CT scan in this study was 6 days (range 1-17 days), ten (25.0\%) participants had less than 3 days of clinical onset before the CT scan. Since the ideal CT time for evaluating possible complications of acute pancreatitis is after 3 days $^{2}$, this could have decreased the accuracy of our CT studies to evaluate the complications and grade the severity of acute pancreatitis in some participants.

Some studies on using CT to predict the severity of acute pancreatitis have been recently published. Meyrignac et al. ${ }^{15}$ reported a significant relationships between an 
extrapancreatic necrosis volume of more than the 100 $\mathrm{mL}$ threshold and clinical severity outcome (organ failure, infection, length of hospital stay, need for intervention, and death); they found a significantly higher area under the receiver operating characteristic curve $(A \cup C)$ than those of the Balthazar score, CTSI, and C-reactive protein. PamiesGuilabert et al. ${ }^{16}$ have proposed the combined pancreatic and extrapancreatic necrosis volume of more than $75 \mathrm{~mL}$ to be a useful marker for the prediction of the severity of acute pancreatitis, even better than the Balthazar score and CTSI. Monreal-Robles et al. ${ }^{17}$ claimed that a peritoneal cavity circumference of greater than 85 centimeters, measured via an abdominal CT scan, was useful in the initial forecast of clinical severity and mortality. In light of this study's findings and those of other studies, there is a need for further studies on the role of CT imaging for predicting the severity of acute pancreatitis.

Given our study's retrospective design, we acknowledge some limitations. First, its sample size was small, and the time between the CT scan and the onset of the disease had considerable variation (1-17 days). Furthermore, the CT techniques employed also varied along the study period. The lack of pancreatic or arterial phase images in some participants' CT scans also played a role in compromising the ability to evaluate pancreatic necroses, pseudoaneurysms, or possible pancreatic masses. Second, two participants who passed away had short durations of hospital stay, which decreased the accuracy of using the length of hospital stay as a clinical outcome. Third, some participants had co-morbid diseases, such as cirrhosis, and some CT features that affected the CTSI and MCTSI interpretation (e.g., ascites, bowel wall edema, etc.). Furthermore, some participants were admitted with a primary diagnosis of acute pancreatitis but developed other systemic complications during admission, which affected their clinical outcome.

\section{Conclusion}

Our study showed moderate and fair agreement between CTSI and MCTSI scores and the clinical severity assessment findings using the determinant-based classification of acute pancreatitis severity, respectively. Patients with high CTSIMCTSI scores (moderate and severe groups) tended to show poorer clinical outcomes compared to those with low scores (mild group).

\section{Conflict of interest}

There are no potential conflicts of interest to declare.

\section{References}

1. Banks PA, Bollen TL, Dervenis C, Gooszen HG, Johnson CD, Sarr MG, et al. Classification of acute pancreatitis--2012: revision of the Atlanta classification and definitions by international consensus. Gut 2013;62:102-11.

2. Thoeni RF. The revised Atlanta classification of acute pancreatitis: its importance for the radiologist and its effect on treatment. Radiology 2012;262:751-64.

3. Tenner S, Baillie J, DeWitt J, Vege SS; American College of Gastroenterology. American College of Gastroenterology guideline: management of acute pancreatitis. Am J Gastroenterol 2013;108:1400-16.

4. Mao L, Qiu Y. The classification of acute pancreatitis: current status. Intractable Rare Dis Res 2012;1:134-7.

5. Dellinger EP, Forsmark CE, Layer P, Lévy P, Maraví-Poma E, Petrov MS, et al. Determinant-based classification of acute pancreatitis severity: an international multidisciplinary consultation. Ann Surg 2012;256:875-80.

6. Vincent JL, Moreno R, Takala J, Willatts S, De Mendonça A, Bruining $\mathrm{H}$, et al. The SOFA (Sepsis-related Organ Failure Assessment) score to describe organ dysfunction/failure. On behalf of the Working Group on Sepsis-Related Problems of the European Society of Intensive Care Medicine. Intensive Care Med 1996;22:707-10.

7. Balthazar EJ, Robinson DL, Megibow AJ, Ranson JH. Acute pancreatitis: value of CT in establishing prognosis. Radiology 1990;174:331-6. 
8. Balthazar EJ. Acute pancreatitis: assessment of severity with clinical and CT evaluation. Radiology 2002;223:603-13.

9. Mortele KJ, Mergo PJ, Taylor HM, Ernst MD, Ros PR. Renal and perirenal space involvement in acute pancreatitis: spiral CT findings. Abdom Imaging 2000;25:272-8.

10. Wiesner W, Studler U, Kocher T, Degen L, Buitrago-Tellez CH, Steinbrich W. Colonic involvement in non-necrotizing acute pancreatitis: correlation of CT findings with the clinical course of affected patients. Eur Radiol 2003;13:897-902.

11. Lankisch PG, Pflichthofer D, Lehnick D. No strict correlation between necrosis and organ failure in acute pancreatitis. Pancreas 2000;20:319-22.

12. Mortele KJ, Wiesner W, Intriere L, Shankar S, Zou KH, Kalantari $\mathrm{BN}$, et al. A modified CT severity index for evaluating acute pancreatitis: improved correlation with patient outcome. Am J Roentgenol 2004;183:1261-5.

13. Bollen TL, Singh VK, Maurer R, Repas K, van Es HW, Banks PA, et al. Comparative evaluation of the modified CT severity index and CT severity index in assessing severity of acute pancreatitis. Am J Roentgenol 2011;197:386-92.

14. Akoglu H. User's guide to correlation coefficients. Turk J Emerg Med 2018;18:91-3.

15. Meyrignac O, Lagarde S, Bournet B, Mokrane FZ, Buscail L, Rousseau $\mathrm{H}$, et al. Acute pancreatitis: extrapancreatic necrosis volume as early predictor of severity. Radiology 2015;276:11928.

16. Pamies-Guilabert J, Del Val Antoñana A, Collado JJ, Rudenko $\mathrm{P}$, Meseguer A. Pancreatic necrosis volume - A new imaging biomarker of acute pancreatitis severity. Eur J Radiol 2020; 130:109193.

17. Monreal-Robles R, Kohn-Gutiérrez AE, Sordia-Ramírez J, Zúñiga-Segura JA, Palafox-Salinas JA, de la Rosa-Pacheco $S$, et al. Peritoneal cavity circumference on computed tomography predicts outcomes in acute pancreatitis. Eur $\mathrm{J}$ Radiol 2020;132:109327. 